\title{
Les moteurs moléculaires du transport axonal
}

Il existe un mouvement incessant de matériel depuis le corps cellulaire du neurone jusqu'à sa terminaison axonale (transport axonal antérograde) et inversement (transport rétrograde). Les molécules sont transportées en association avec des vésicules qui « glissent » le long des microtubules, à la vitesse de $400 \mathrm{~mm} /$ jour dans le sens antérograde et de $200 \mathrm{~mm} /$ jour dans le sens rétrograde. Les enzymes catalysant ces déplacements sont probablement des ATPases, équivalents fonctionnels de la myosine musculaire et de la dynéine des cils et flagelles. Deux de ces protéines, intervenant dans le transport axonal et probablement dans d'autres processus de motilité intracellulaire, ont été caractérisées in vitro: une enzyme soluble, la kinésine, et une protéine de la famille des protéines capables de copolymériser avec la tubuline pour former des microtubules, la MAP 1 C.

\section{Ghislaine Filliatreau Chargée de recherche à l'Inserm}

\section{RÉFÉRENCES}

1. Allen RD, Allen NS, Travis JL. Videoenhanced differential interference contrast (AVEC-DIC) microscopy: a new method capable of analyzing microtubule related movement in the reticulopodial network of Allogromia laticollaris. Cell Motil 1981; 1 : 291-302.

2. Allen RD, Weiss DG, Hayden JH, Brown DT, Fujiwake H, Simpson M. Gliding movement of and bidirectional organelle transport along single native microtubules from squid axoplasm: evidence for an active role of microtubules in cytoplasmic transport. J Cell Biol 1985 ; 100 : 1736-52.

\section{ADRESSE}

G. Filliatreau : service hospitalier Frédéric Joliot, hôpital d'Orsay, 91406, Orsay, France.

\section{TIRÉS A PART}

G. Filliatreau: service hospitalier Frédéric Joliot, hôpital d'Orsay, 91406, Orsay, France. $\mathrm{m} / \mathrm{s} n^{\circ} 6$ vol. 4 , juin 88 e neurone est une cellule très régionalisée, dans laquelle les synthèses protéiques sont confinées au corps cellulaire. C'est également une cellule très asymétrique, où le volume de l'axone représente typiquement plusieurs milliers de fois celui du corps cellulaire. Le maintien et le fonctionnement de l'axone et de sa terminaison nécessitent donc un ravitaillement massif et permanent, assuré par le transport axonal antérograde, lent et rapide. De plus, les macromolécules qui n'ont pas été sécrétées ou qui ont été captées à la terminaison peuvent être ramenées vers le corps cellulaire par le transport rétrograde.

Le transport axonal est donc vital pour le fonctionnement du système nerveux; il constitue en outre un modèle privilégié pour l'étude de la motilité intracellulaire. En effet, de tels déplacements de molécules nouvellement synthétisées ou à recycler existent dans toutes les cellules, même s'ils ne sont généralement pas aussi visibles.

\section{Transport antérograde lent et rapide}

Le transport antérograde lent assure le renouvellement de $80 \%$ des pro- téines totales. Il transporte essentiellement des protéines de structure (cytosquelette) et des enzymes du métabolisme intermédiaire, à des vitesses de 1 à $4 \mathrm{~mm} /$ jour. Son mécanisme est encore inconnu.

Le transport antérograde rapide véhicule, à des vitesses d'environ $400 \mathrm{~mm} /$ jour, des vésicules et des protéines membranaires, cependant que le transport rétrograde, également rapide $(200 \mathrm{~mm} /$ jour$)$, ramène vers le corps cellulaire des vésicules de plus grande taille.

Ces deux transports nécessitent la présence d'ATP et sont bloqués par des antimitotiques connus pour désorganiser les microtubules, comme la colchicine ou la vinblastine. Les caractéristiques de ces transports, jointes à la présence de nombreux microtubules longitudinaux dans les axones, ont très tôt orienté les recherches vers la mise en évidence d'une ATPase qui serait, comme la dynéine des cils et flagelles, capable de se lier aux microtubules pour générer du mouvement (voir encart, page 376). Malgré la mise en évidence de plusieurs ATPases ressemblant à la dynéine dans certaines cellules (œufs d'échinodernes, algues unicellulaires), rien de précis n'avait permis de valider cette hypothèse «moléculaire» d'une dynéine capa- 
ble de travailler dans le cytoplasme des cellules, et d'autres modèles du transport axonal rapide restaient tout à fait plausibles (micro-courants intracytoplasmiques, transports intramembranaires).

C'est en 1981 qu'un premier pas décisif est franchi par Allen qui, en perfectionnant les techniques vidéo appliquées à la microscopie optique, observe pour la première fois en direct, dans une cellule vivante, le déplacement de myriades de petites vésicules le long des microtubules [1].

\section{Un modèle cellulaire de transport axonal}

Très vite Allen va mettre en évidence le même phénomène dans l'axone géant de calmar, un matériel particulièrement favorable car on peut en extruder* l'axoplasme: on obtient ainsi un cylindre de cytoplasme débarrassé de sa gaine membranaire, mais qui garde sa structure et sa capacité de transport même après plusieurs heures d'incubation. C'est à partir de ce matériel qu'on peut, pour la première fois, analyser précisément le transport bidirectionnel, rapide et continu, des vésicules sur des microtubules bien individualisés [2, 4, 13]. On s'aperçoit, en étalant l'axoplasme sur une lame de verre, que des fragments de microtubules isolés peuvent glisser à la surface du verre, traversant rapidement le champ du microscope tout en continuant à transporter des vésicules de rencontre (figure 1, p. 375).

Pendant que ces observations sur les microtubules natifs se poursuivent, plusieurs équipes entreprennent de reconstituer le système in vitro. On montre rapidement que des vésicules isolées depuis l'axone géant de calmar sont capables, en présence d'ATP, de glisser sur des microtubules reconstitués à partir de tubuline purifiée et déposés sur une lame de verre. Si l'on a joute à la préparation un extrait soluble d'axoplasme, on augmente considérablement la quantité de vésicules transportées et on induit un déplacement des microtubules très semblable à celui observé précédemment.

Ce déplacement des microtubules sur lame de verre va être utilisé comme facteur soluble responsable du mouvement. En fait, le travail sera grandement facilité par le fait qu'on peut induire une association stable entre microtubules et vésicules si l'on incube l'axoplasme en présence de 5 ' adénylyl imidiphosphate (AMP PNP), un analogue non hydrolysable de l'ATP. L'AMP-PNP est un ligand compétitif de l'ATP qui, en se liant à la protéine de transport portée par les vésicules, augmente considérablement leur affinité pour les microtubules; en l'absence d'hydrolyse de l'AMP-PNP, le complexe ainsi formé ne se dissocie pas.

Dans ces conditions, on piège la protéine de transport et les vésicules sur les microtubules qu'on isole par centrifugation. En ajoutant de l'ATP aux microtubules obtenus, on provoque le relargage des vésicules et la solubilisation d'une ATPase soluble, la kinésine

\section{La kinésine}

Chez les mammifères, la kinésine apparaît constituée de deux types de sous-unités, de 120 et $62 \mathrm{kDa}^{* *}$, mais sa structure quaternaire n'est pas connue. Son activité ATPasique est stimulée par la présence de microtubules, mais elle reste faible par rapport à celles de la dynéine et de la myosine. Les inhibiteurs spécifiques de la dynéine sont relativement sans effets sur elle : la kinésine apparaît, au total, assez différente de la protéine de type dynéine recherchée au départ [3, 15].

De nombreuses questions restent à résoudre quant à son rôle dans le transport rapide antérograde. En présence d'AMP-PNP, la kinésine purifiée est capable d'induire une association stable entre microtubules purifiés et vésicules. Cependant, si on ajoute de l'ATP à la préparation, on observe un relargage des vésicules mais pas de transport: il semble que d'autres facteurs soient nécessaires

* On extrude l'axoplasme de l'axone géant de calmar en comprimant progressivement l'axone, comme on fait sortir la pâte d'un tube dentifrice.

* kDa: kilodalton. pour «convertir» l'hydrolyse de l'ATP en déplacement [14].

Dans les modèles de mécanisme envisagés, la kinésine constituerait les «bras » observés in vivo entre microtubules et vésicules [9], même si la faible affinité in vitro de la protéine purifiée par les uns et les autres reste à expliquer.

De même, sa faible activité ATPasique ne semble pas suffisante pour rendre compte des vitesses de déplacement observées. Cependant on peut isoler la protéine en présence de tripolyphosphate (PPPi), un autre analogue non hydrolysable de l'ATP. Il semble qu'on obtienne alors une protéine dont l'activité ATPasique - mesurée en présence d'ATP - est plus élevée que celle de la kinésine isolée en présence d'AMP-PNP et surtout, plus efficacement stimulée par la présence de microtubules [8]. Là encore, on peut penser que les deux préparations diffèrent par la présence de cofacteurs qui interviennent, in vivo, pour contrôler l'activité ATPasique de la protéine.

\section{Transport rétrograde}

Les microtubules possèdent une polarité interne, dictée par l'orientation des dimères de tubuline et repérée par le fait qu'une de leurs extrémités [notée $(+)$ ] est plus favorable que l'autre [notée (-)] pour la polymérisation. On a vu, in vivo, dans l'axoplasme extrudé, que des vésicules parcourent dans les deux sens chaque microtubule.

Cependant, les expériences faites in vitro avec la kinésine purifiée ont montré que celle-ci ne déplace les vésicules que dans un sens, de l'extrémité $(-)$ vers l'extrémité $(+)$ des microtubules. Dans l'axone, où les microtubules sont orientés de manière unipolaire, telle que leur extrémité $(+)$ soit distale par rapport au corps cellulaire du neurone [5], le mouvement provoqué par la kinésine doit être unidirectionnel et antérograde. La recherche d'un facteur responsable du transport rétrograde a donc été entreprise [16] mais ce n'est que très récemment que Paschal et al. ont isolé une APTase capable de se lier aux microtubules et de provoquer leur déplacement dans le sens inverse de celui observé avec la kiné- 


\section{RÉFÉRENCES}

3. Brady ST. A novel brain ATPase with properties expected for the fast axonal transport motor. Nature 1985 ; 317 : 73-5.

4. Brady ST, Lasek R J, Allen RD. Videomicroscopy of fast axonal transport in extruded axoplasm : a new model for study of molecular mechanisms. Cell Motil 1985 ; 99 : 81-101.

5. Filliatreau G, Di Giambernardino L. Microtubule polarity in myelinated axons as studied after decoration with tubulin. Biol Cell $1981 ; 42$ : 69-72.

6. Hollenbeck PJ, Chapman K. A novel microtubule associated protein from mammalian nerve shows ATP-sensitive binding to microtubules. J Cell Biol 1986; 103 : 1539-45.

7. Koszka Ch, Foisner R, Seyfert HM, Wiche $\mathrm{G}$. Isolation of a Ca-protease resistant high Mr microtubule binding protein from mammalian brain: characterization and properties partially expected for a dynein-like molecule. Protoplasma 1987 ; 138 : 54-61.

8. Kuznetsov SA, Gelfand VI. Bovine brain kinesin is a microtubule activated ATPase. Proc Natl Acad Sci USA 1986; 79 : 2603-7.

9. Miller RH, Lasek RJ. Cross bridges mediate anterograde and retrograde vesicle transport along microtubules in squid axoplasm. J Cell Biol 1985; 101 : 2181-93.

10. Paschal BM, Shpetner HS, Vallee RB. MAP IC is a microtubule activated ATPase which translocates microtubules in vitro and has dynein-like properties. J Cell Biol 1987; $105: 1273-82$.

11. Paschal BM, Vallee RB. Retrograde transport by the microtubule-associated protein MAP 1C. Nature $1987 ; 330: 181-3$.

12. Pratt MM. Stable complexes of axoplasmic vesicles and microtubules : protein composition and ATPase activity. J Cell Biol 1986 ; 103 : 957-68.

13. Schnapp BJ, Vale RD, Sheetz MP, Reese TS. Single microtubules from squid axoplasm support bidirectional movement of organelles. Cell 1985; 40 : 455-62.

14. Schroer TA, Sheetz MP. Identification of kinesin-binding proteins in squid brain. $J$ Cell Biol 1986 ; 103 : 553a.

15. Vale RD, Reese TS, Sheetz MP. Identification of a novel force generating protein, kinesin, involved in microtubule based motility. Cell $1985 ; 42$ : $39-50$.

16. Vale RD, Schnapp BJ, Mitchison $T$, Steuer E, Reese TS, Sheetz MP. Different axoplasmic proteins generate movement in opposite directions along microtubules in vitro. Cell $1985 ; 43$ : 623-32.

$\mathrm{m} / \mathrm{s} n^{\circ} 6$ vol. 4 , juin 88

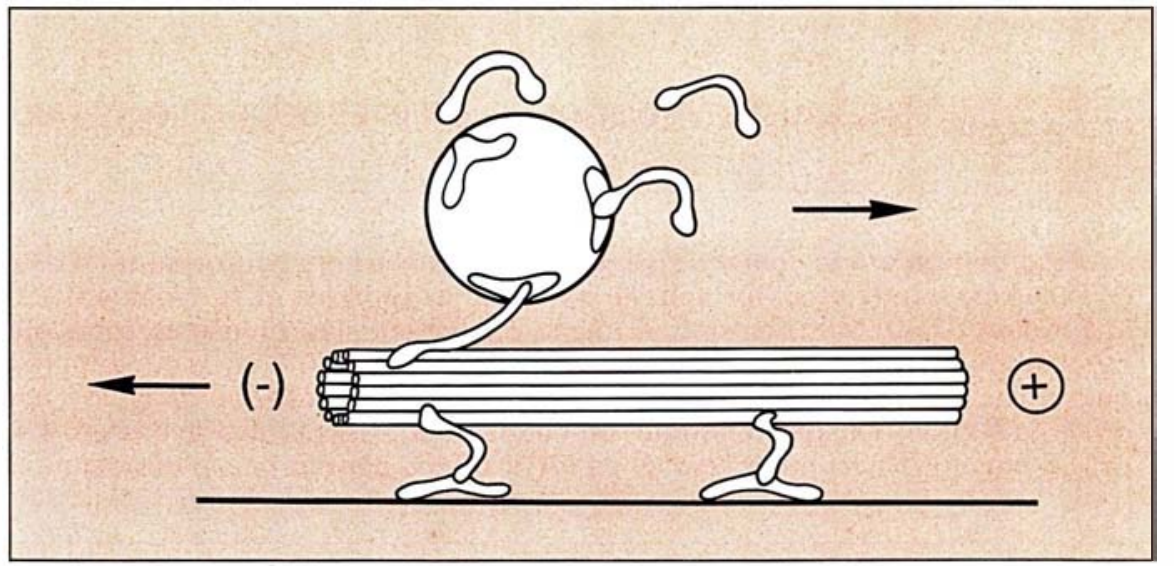

Figure 1. Modèle hypothétique du mouvement des vésicules et des microtubules in vitro (mouvement des vésicules sur le microtubule, et des microtubules surla lame de verre). C'est la kinésine absorbée à la surface d'une lame de verre négativement chargée qui produit le glissement des microtubules purifiés. De même, la kinésine absorbée sur des microbilles de latex portant des charges négatives permet leur transport orienté le long des microtubules. In vivo, la kinésine pourrait se lier aux vésicules axoplasmiques par l'intermédiaire d'une protéine de membrane. La direction du mouvement généré par la kinésine in vitro se fait, pour les vésicules et les microbilles, de l'extrémité (-) vers l'extrémité(t) du microtubule, c'est-à-dire en sens opposé au mouvement du microtubule purifié sur la lame de verre.

sine $[10,11]$. Une première surprise est que cette protéine, loin d'être inconnue comme l'était la kinésine, a été identifiée à l'une des HMW. MAPS (high molecular weight, microtubule-associated protein), c'est-à-dire à l'une des protéines de haut poids moléculaire capables de copolymériser avec la tubuline pour former des microtubules. La seconde surprise est que cette kinésine rétrograde, la MAP-1C, possède bien les caractéristiques de type dynéine qu'on cherchait au départ (tableau I, p. 376). Le déplacement des microtubules sur la lame de verre est, en présence de cette protéine, quatre fois plus rapide que celui induit par la kinésine. Comme la kinésine, la MAP-1C est enrichie dans une fraction de microtubules purifiés en l'absence de nucléotides hydrolysables, et elle est massivement solubilisée quand on ajoute de l'ATP à la préparation.

La MAP-1C et la kinésine ont relancé la recherche des moteurs moléculaires du type dynéine ou myosine. On dispose maintenant de la possibilité d'observer directement par vidéomicroscopie les propriétés translocatrices des protéines, et également d'une meilleure connaissance des stratégies à utiliser pour «piéger » des ATPases sur les microtubules. Ainsi, plusieurs ATPases possédant des propriétés de type dynéine et/ou kinésine ont été isolées à partir de tissu nerveux mais leur fonction dans le transport axonal n'a pas pu être clairement établie [6, 7, 12]. A l'inverse, la kinésine et d'autres ATPases ont été mises en évidence dans différentes cellules non neuronales où elles jouent évidemment d'autres rôles. Ainsi, les résultats obtenus dans l'axone ont permis de proposer des modèles pour des processus aussi généraux que les déplacements des chromosomes au cours de la mitose ou les mouvements du réticulum endoplasmique dans les cellules et plusieurs équipes s'emploient actuellement à reconstituer ces systèmes in vitro. Parallèlement, l'obtention d'anticorps monoclonaux et de sondes génétiques permettra d'explnrer les fonctions de ces protéines.

La recherche de «moteurs enzymatiques» assurant les mouvements intracellulaires liés aux microtubules (et aux microfilaments), à nouveau très active, soulève pour l'instant plus de questions qu'elle n'apporte de réponses. Gageons cependant que les résultats vont maintenant se multiplier, dans un domaine où les choses avancent vite! 
Tableau 1

TABLEAU PROVISOIRE DES PROPRIÉTÉS DE DIFFÉRENTS * MOTEURS ENZYMATIQUES *

Tableau comparant les caractéristiques des protéines qui hydrolysent l'ATP pour produire du mouvement. Les deux ATPases les mieux étudiées sont la myosine des muscles et la dynéine des flagelles, deux organes spécialisés dans la production du mouvement. II existe également des myosines intracellulaires qui sont, comme les dynéines cytoplasmiques. assez mal connues. La kinésine et la MAP-1C semblent présentes dans une large variété de cellules qui, à la différence du neurone, ne sont pas spécialisées dans les transports intracellulaires. Le NEM (Néthylmaléimide) est un inhibiteur de l'activité ATPasique des dynéines. L'AMP-PNP (5' adénylyl-imidiphosphate) est un analogue non hydrolysable de l'ATP qui ne permet pas la dissociation du complexe microtubule-kinésine.

\begin{tabular}{|c|c|c|c|c|c|}
\hline & $\begin{array}{l}\text { Myosine } \\
\text { (muscle) }\end{array}$ & $\begin{array}{l}\text { Dynéine } \\
\text { (flagelles) }\end{array}$ & $\begin{array}{c}\text { Dynéine } \\
\text { (cytoplasmique) }\end{array}$ & Kinésine & MAP IC \\
\hline Substrat & microfilaments & microtubules & microtubules & microtubules & microtubules \\
\hline $\begin{array}{l}\text { Direction } \\
\text { du transport }\end{array}$ & vers le bout + & vers le bout + & vers le bout + & $\begin{array}{l}\text { vers le bout }+ \\
\text { (antérograde } \\
\text { dans l'axone) }\end{array}$ & $\begin{array}{l}\text { vers le bout - } \\
\text { (rétrograde } \\
\text { dans l'axone) }\end{array}$ \\
\hline $\begin{array}{l}\text { Coefficient } \\
\text { de sédimentation }\end{array}$ & $6 \mathrm{~S}$ & $21 \mathrm{~S}$ à $30 \mathrm{~S}$ & $\simeq 20 \mathrm{~S}$ & $9,6 \mathrm{~S}$ & $20 \mathrm{~S}$ \\
\hline $\begin{array}{l}\text { PM } \\
\text { des polypeptides }\end{array}$ & 200 & $\simeq 450$ (oursin) & $\simeq 350$ & $2 \times 110 ; 1 \times 70$ & $\simeq 300$ \\
\hline $\begin{array}{l}\text { Inhibition } \\
\text { vanadate }\end{array}$ & - & + & + & - & + \\
\hline $\begin{array}{l}\text { Inhibition } \\
\text { NEM }\end{array}$ & + & + & + & - & + \\
\hline $\begin{array}{l}\text { Vitesse } \\
(\mu \mathrm{m} / \mathrm{sec})\end{array}$ & $2-5$ & 10 & 2 & 0,5 & 2 \\
\hline $\begin{array}{l}\text { Inhibition } \\
\text { AMP-PNP }\end{array}$ & - & - & - & + & + \\
\hline
\end{tabular}

\section{LES PROTÉINES UTILISANT L'ÉNERGIE D'HYDROLYSE DE l'ATP POUR PRODUIRE DU MOUVEMENT}

Jusqu'à très récemment, on ne connaissait que deux "moteurs moléculaires" des mouvements: la myosine, qui participe à la contraction musculaire, et la dynéine qui produit les battements des cils et des flagelles. Malgré de nombreuses différences (tableau I), les deux protéines travaillent probablement de la même manière, faisant glisser deux filaments l'un par rapport à l'autre, par un cycle d'association - dissociation utilisant l'hydrolyse de l'ATP. Toutes deux sont formées de têtes globulaires portant chacune un site de liaison pour le filament et un site de liaison pour l'ATP, attachées ensemble par une tige plus ou moins flexible.

Le cycle du mouvement peut être schématisé ainsi: le complexe filament-enzyme est dissocié très rapidement par fixation d'ATP; il y a ensuite hydrolyse plus lente de l'ATP resté fixé à l'enzyme, puis réassociation de l'enzyme avec le filament avec dissociation des produits de l'hydrolyse, donc possibilité de fixer à nouveau l'ATP. Le cycle de la kinésine pourrait être différent, puisqu'un analogue non hydrolysable de l'ATP ne permet pas la dissociation: il faut supposer que l'hydrolyse de l'ATP intervient dans cette dissociation. 


\section{Summary}

In neurons, many proteins are transported between the cell body and the axon terminal in association with membranous organelles, which move at rates of up to $5 \mu \mathrm{m} / \mathrm{sec}$ in both the anterograde (toward the terminal) and retrograde (toward the cell body) directions. Recently, in vivo observations and in vitro models of fast axonal transport have been developed, allowing to describe two microtubule-based motility proteins. The anterograde motor would be a soluble, microtubule activated ATPase called kinesin, which generates in vitro the gliding of microtubules on glass and the transport of vesicles on microtubules. Unexpectedly, this protein is structurally and enzymatically different from dynein, the only ATPase known to generate movement using microtubules as a substrate. By contrast MAP-1C, the putative retrograde motor, can be considered as a dynein-like protein, providing the long-awaited validation of dyneins as cytoplasmic motors of intracellular transports. Nevertheless, many uncertainties remain about the detailed mechanism of the rapid axonal transport.

a BRÈVES

Un premier essai humain d'un vaccin antipalustre. Le paludisme continue de représenter l'un des fléaux les plus coûteux en vie humaine dans les pays tropicaux en voie de développement. Il serait responsable d'un quart de la mortalité infantile (1 à 4 ans) en Afrique. Une équipe colombienne [1] a identifié les antigènes majeurs des formes asexuées (érythrocytaires) de Plasmodium falciparum et déterminé, après séparation électrophorétique des protéines dénaturées, lesquelles, injectées à des singes du nouveau monde sensibles au parasite, stimulaient le développement d'une immunité protectrice. La séquence partielle en acides aminés de certaines de ces protéines a été déterminée, ce qui a permis de synthétiser plusieurs oligopeptides qui, utilisés à leur tour pour immuniser les animaux d'expérience, se sont révélés protecteurs. Patarroyo et al. [1] ont alors synthétisé deux peptides plus grands ( 45 résidus) reproduisant chacun la séquence de trois des oligopeptides «protecteurs», et les ont polymérisés en deux protéines de grande taille (100 et $150 \mathrm{kDa})$ qui ont ensuite été testées chez 13 volontaires non impaludés, membres des forces armées. La vaccination elle-même se révéla sans effets secondaires notables. Avec l'une des protéines synthétiques, quatre des cinq volontaires vaccinés puis infectés furent très efficacement protégés, avec une augmentation retardée, lente et spontanément résolutive de la parasitémie. Aucune relation nette ne put être établie entre la protection et la réponse immune, appréciée par analyse des anticorps et de la réactivité cellulaire. Ces résultats, encore très préliminaires, représentent le premier essai humain d'une vaccination contre les stades érythrocytaires (mérozoïtes, schizontes) de P. falciparum.

[1. Pattaroyo ME, et al. Nature 1988 ; 332: 158-61.]

Chez les oiseaux, une différence sexuelle dans la reconnaissance de l'autre. Le chant des oiseaux est très précisément contrôlé et varie peu, au sein d'une espèce, d'un animal à l'autre. Il joue en effet un rôle essentiel dans la reconnaissance entre les animaux d'une même espèce. Pour le mâle qui a à défendre son territoire contre l'intrusion d'un rival, ce qui est essentiel, c'est d'être mis en alerte dans tous les cas. Un excès de précaution n'étant pas nuisible, le merle à ailes rouges mâle sera mis ainsi en alerte même par un chant un peu différent de celui de l'espèce. Pour la femelle, au contraire, il importe avant tout de distinguer le chant du fécondateur éventuel de celui d'un oiseau d'une autre espèce ; cette exigence de discrimination peut conduire ainsi l'oiselle à négliger un congénère enroué sans que cela porte à conséquence, les occasions ne manquant pas plus chez les merles que dans les autres espèces. De fait, des expériences consistant à observer le déclenchement par des chants d'oiseau d'un comportement nuptial chez des merles à ailes rouges femelles préalablement stimulées par les oestrogènes, ont démontré que celui-ci n'apparaissait qu'après audition d'un chant répondant intégralement aux canons en vigueur dans l'espèce. Peut-être la différence sexuelle dans la reconnaissance de l'autre, via le chant ou d'autres perceptions, estelle un phénomène général ?

[Searcy WA, Brenowitz EA, Nature 1988 ; 332 : 152-4.]

La substance amyloïde des îlots pancréatiques (IAPP) a été localisée à l'aide de divers antisérums. Elle est mise en évidence dans les îlots de chat, chien, souris et rat, mais non chez le cheval et le veau. L'IAPP est située dans les cellules $\beta$ qui sécrétent l'insuline, localisée à la partie externe des granules de sécrétion alors que l'insuline se trouve dans la partie centrale. Ces résultats suggèrent que l'IAPP ou son précurseur est synthétisée par les cellules $\beta$ pancréatiques et stockée dans les granules en vue d'une co-sécrétion avec l'insuline.

[Johnson KH, et al. Am J Path 1988; $130: 1-8$. 